\title{
METÁSTASIS HEPÁTICAS DE ORIGEN COLORRECTAL SINCRÓNICAS: ¿INTERVENCIÓN SIMULTÁNEA O SECUENCIAL?*
}

\author{
Drs. Beatriz Pérez-Cabrera ${ }^{1}$, Antonio Palomeque-Jiménez ${ }^{1}$, Patricia Navarro-Sánchez ${ }^{2}$, \\ Amanda Rocío González-Ramírez ${ }^{3}$, Francisco Navarro-Freire ${ }^{1}$ \\ 1 Unidad de Cirugía Hepatobiliopancreática. \\ 2 Servicio de Radiodiagnóstico. \\ 3 Fundación Pública andaluza para la investigación biosanitaria de Andalucía oriental (FIBAO). \\ Hospital Universitario San Cecilio. \\ Granada, España.
}

\begin{abstract}
\section{Simultaneous or sequential treatment of liver metastases of colon cancer?} Experience in 76 patients

Background: Synchronous liver metastases of colon cancer can be managed with sequential or simultaneous surgical management of the primary tumor and the metastases. Aim: To compare the evolution of patients whose liver metastases were treated sequentially or simultaneously. Material and Methods: Retrospective analysis of 76 patients aged $63 \pm 11$ years (67\% males). In 25, metastases were managed simultaneously and in 51 there were treated sequentially after a period of chemotherapy. All interventions were performed by the same surgeon. Results: Patients treated sequentially had a higher number of metastases and more lymph nodes involved than their counterparts treated simultaneously. The overall resectability index was $78 \%$. Eighteen major and 28 minor hepatic resections were carried out. Significantly more major resections were carried out in the sequential treatment group. Mean hospital stay was 11 days and $20 \%$ of patients had complications, with no differences between groups. Survival at one, three and five years was 75, 45 and 36\% in the simultaneous treatment and 76, 49 and $29 \%$ in the sequential treatment group (with no significant differences between groups). Conclusions: In this group of patients no differences in complications or survival were observed when liver metastases were treated simultaneously or sequentially. However groups were not homogeneous.
\end{abstract}

Key words: Liver metastases, colon cancer, surgical treatment.

\section{Resumen}

Introducción: Existen distintas estrategias para el tratamiento de las metástasis hepáticas de origen colorrectal sincrónicas (MHCRS): cirugía secuencial, según respuesta a quimioterapia, intervención simultá-

*Recibido el 11 de junio de 2014 y aceptado para publicación el 14 de julio de 2014.

Los autores no refieren conflictos de interés.

Correspondencia: Dra. Beatriz Pérez-Cabrera bperezcabrera@ugr.es 
nea del tumor y las metástasis o cirugía hepática previa al tumor primario; el uso de una u otra estrategia es aún controvertido. Objetivo: Comparar la morbimortalidad y supervivencia en dos grupos de pacientes con MHCRS intervenidos de forma simultánea versus secuencial. Pacientes y Métodos: Definimos las MHCRS como aquellas que se diagnostican antes o durante la intervención del tumor primario. Se comparan dos grupos de pacientes con MHCRS, 25 sometidos a intervención simultánea (grupo 1) y 51 tras quimioterapia (intervención secuencial: grupo 2). La cirugía hepática la realizó el mismo cirujano. Revisamos datos del paciente, del tumor primario, intervención quirúrgica, transfusión perioperatoria, morbimortalidad y supervivencia. Resultados: 76 pacientes, con edad media de 62,79 $\pm 11,3$ años. El número de metástasis y la invasión ganglionar del tumor primario fueron mayores en el grupo 2 de forma estadísticamente significativa. Índice de resecabilidad: 77,6\%: 18 resecciones hepáticas mayores y 28 menores, con diferencias significativas entre ambos grupos $(p=0,05)$. La estancia media $(10,89$ días $)$, Índice de morbilidad $(19,7 \%)$ y supervivencia actuarial a 1,3 y 5 años fueron similares $(75 \%, 45 \%$ y $36 \%$ en el grupo 1 y de $76 \%, 49 \%$ y $29 \%$ en el grupo 2$)$. Mortalidad: 1,6\%. Conclusiones: Las MHCRS pueden ser intervenidas de forma simultánea al tumor primario en pacientes seleccionados siempre que el equipo sea especializado. La morbimortalidad y la supervivencia son similares tanto en la intervención simultánea como en la secuencial.

Palabras clave: Metástasis hepáticas sincrónicas, cáncer colorrectal, hepatectomía.

\section{Introducción}

Las metástasis hepáticas $(\mathrm{MH})$ ocurren entre un 5 y un $40 \%$ de los pacientes con carcinoma colorrectal; un $15-25 \%$ se presentarán de forma sincrónica (MHCRS) y un $25-40 \%$ serán metacrónicas ${ }^{1}$. La resección quirúrgica es el único tratamiento potencialmente curativo y, en el caso de las metástasis sincrónicas, se debate si se deben resecar simultáneamente, antes o después del tumor primario; hasta ahora, la resección por etapas o secuencial (primario-quimioterapia-metástasis), ha sido el gold estándar para las MHCRS entendiendo que la cirugía simultánea presentaba altas tasas de morbimortalidad. Actualmente aumentan los grupos que optan por una cirugía simultánea del primario y de las metástasis si se cumplen algunas condiciones: que no haya enfermedad extrahepática; que las metástasis puedan ser resecadas por el mismo abordaje que el tumor primario; que se pueda hacer una resección R0 dejando un volumen aceptable de hígado sano; que el cirujano sea experto y que el paciente no presente comorbilidad importante ${ }^{2,3}$. Existen publicaciones en las que la morbimortalidad es mayor en la cirugía simultánea ${ }^{4,5}$, en tanto que otras obtienen resultados similares con ambas estrategias $^{5-7}$. El objetivo de este trabajo es evaluar la morbimortalidad y supervivencia de los pacientes con MHCRS intervenidos de forma simultánea con el tumor primario y aquellos que fueron intervenidos de forma secuencial, previa quimioterapia.

\section{Pacientes y Métodos}

Definimos las MHCRS en este estudio, como aquellas diagnosticadas antes o durante la resección del tumor primario. Todos los pacientes han sido evaluados por un equipo multidisciplinario en el que se sigue un protocolo para el tratamiento de las MH, que ha cambiado a lo largo de los años: hasta 2005, las MH se trataban después de haber resecado el tumor primario y recibido quimioterapia. Posteriormente, se consideró la intervención simultánea siempre que el número de metástasis fuera $\leq$ de 3 y de localización abordable por la misma incisión para ambos procedimientos.

Hemos realizado un estudio retrospectivo comparativo entre dos grupos de pacientes con MHCRS, intervenidos en el Hospital Universitario San Cecilio de Granada, desde marzo de 2003 a junio de 2013. El grupo 1 incluye a los pacientes intervenidos de MH simultáneamente con el tumor primario $(n=25)$ y el grupo 2 a los intervenidos de forma secuencial, tras resección del primario y quimioterapia $(n=51)$. En este último grupo, hay 35 pacientes con un número de metástasis $\leq 3$, que no se han intervenido de forma simultánea por venir de otros hospitales con el primario resecado, o ser anteriores al 2005. También hemos comparado la supervivencia de este sub-grupo (al que hemos denominado a efectos prácticos grupo 3) con el grupo 1 .

La información se ha recogido de una base de datos diseñada en 2000 en la que se incluyeron todos los datos de forma prospectiva. Hemos revisado datos epidemiológicos, localización del tumor primario, tamaño y número de metástasis, marcadores tumorales, intervención quirúrgica, transfusión intraoperatoria, morbimortalidad, estancia hospitalaria y estado actual de los pacientes.

El diagnóstico por imagen de las metástasis se realizó con ecografía y tomografía axial computarizada (TC) y tomografía por emisión de positrones (PET) en pacientes dudosos o con sospecha de recurrencia. 
Los pacientes del grupo 2 fueron tratados con quimioterapia neoadyuvante después de resecar el tumor primario.

La intervención hepática la realizó el mismo cirujano en todos los grupos. La incisión en las intervenciones secuenciales ha sido siempre subcostal en tanto que en las sincrónicas ha variado dependiendo de la localización del tumor primario. Denominamos resección mayor si se han resecado más de 3 segmentos hepáticos ${ }^{8}$. Las complicaciones postoperatorias se han descrito siguiendo los criterios de Clavien-Dindo 9 .

El análisis se realizó con el programa IBMSPSS Versión 21, aplicándose pruebas estadísticas paramétricas ( $\chi^{2}$ y T de Student) y no paramétricas (Mann-Whitney y Kruskal-Wallis), dependiendo del tamaño muestral y del tipo de variable. El análisis de supervivencia fue estimado usando el método de Kaplan-Meier, la significación estadística entre los tiempos de supervivencia se determinó con el test de Log-Rank. Se ha considerado significativo un valor de $\mathrm{p} \leq 0,05$.

\section{Resultados}

Los resultados de nuestro estudio se exponen en la Tabla 1.

\section{Pacientes y características del tumor primario}

En los últimos 10 años se han realizado en nuestro centro 225 intervenciones sobre metástasis hepáticas y de ellas se han extraído los 76 pacientes con MHCRS objeto del estudio, 25 intervenidos simultáneamente $(32,8 \%)$ y 51 de forma secuencial $(67,2 \%)$. La edad media global fue de 62,8 años \pm 11,3 ; el $67 \%$ fueron hombres. No encontramos diferencias estadísticamente significativas entre ambos grupos en cuanto a la edad, sexo, riesgo anestésico (ASA), localización o tamaño del tumor primario, ni valor del antígeno carcinoembrionario (CEA). Sin embargo, sí encontramos diferencias en la invasión nodal que fue significativamente mayor en el grupo $2(\mathrm{p}=0,02)$.

\section{Metástasis y tratamiento quirúrgico}

El número de metástasis osciló entre 1 y 10 , siendo mayor de forma significativa $(p=0,05)$ en el grupo 2.

El índice de resecabilidad global fue de 77,6\%. Se realizaron 18 resecciones hepáticas $(\mathrm{RH})$ mayores y 28 menores, siendo las resecciones mayores más frecuentes en el grupo de cirugía secuencial con una diferencia estadísticamente significativa $(\mathrm{p}=0,05)$; en 13 pacientes se combinó $\mathrm{RH}$ y radiofrecuencia $(\mathrm{RF})$ y en otros 13 se realizó una ablación con RF sola o combinada con embolización portal (3 casos). Todos los pacientes con resección hepática (mayor o menor) tienen margen quirúrgico negativo en el estudio histológico.

En 4 pacientes sólo se realizó exploración hepática sin ningún otro gesto, por metástasis múltiples y afectación extrahepática no sospechada. Tres pacientes fueron sometidos a una segunda resección hepática, 2 a una tercera y 1 a una cuarta. Se transfundieron 10 pacientes del grupo 1 y 25 del grupo 2 .

\section{Morbimortalidad}

Se complicaron 15 pacientes, 7 del grupo 1, destacando una colección en Douglas por fallo de la anastomosis colorectal que precisó reintervención y 8 en el grupo 2, entre las que hubo una colección subhepática drenada por punción radioguiada. El resto de las complicaciones fueron 2 infecciones de herida, 2 flebitis de la vía central, un cuadro de demencia, 5 cuadros de anemia que precisaron transfusión, una fístula colónica, un derrame pleural y una disfunción cardiorespiratoria y renal (Tabla 2).

Un paciente del grupo 1 de 83 años, al que se realizó hemicolectomía derecha y segmentectomía IVb, falleció por insuficiencia cardíaca congestiva (1,3\%).

\section{Supervivencia}

La mediana de supervivencia para el grupo 1 fue de 40,66 meses (rango 0-97) y para el grupo 2 de 34,05 meses (rango 1-154). La supervivencia actuarial a uno, tres y cinco años es de $75 \%$, 45\% y $36 \%$ en el grupo 1 y de $76 \%, 49 \%$ y $29 \%$ en el grupo 2 , sin diferencias entre ambos grupos. El tiempo medio libre de enfermedad fue de 10,18 $\pm 1,4$ meses.

Las curvas de supervivencia quedan reflejadas en la Figura 1 (curva de supervivencia de toda la serie), Figura 2 (curvas de supervivencia de los grupos 1 y 2 ) y Figura 3, en la que hemos representado la supervivencia del grupo 1 frente al grupo 3 (pacientes del grupo 2 con número de $\mathrm{MH} \leq 3$ ) y, como se observa, son prácticamente superponibles. Los 4 pacientes a los que sólo les realizamos una laparotomía exploradora, no fueron incluidos en el análisis de supervivencia entendiendo que, realmente, no se había realizado ningún gesto quirúrgico sobre las $\mathrm{MH}$, pero sí en el resto de parámetros descriptivos por entender que la laparotomía exploradora ya es en sí misma una intervención que puede conllevar morbi-mortalidad.

\section{Discusión}

El concepto de metástasis sincrónica es diferente según autores; algunos, las definen como aquellas que se diagnostican junto al tumor primario ${ }^{2,10,11}$ 
Tabla 1. Resultados en los grupos 1 y 2 y diferencias estadísticas

\begin{tabular}{|c|c|c|c|}
\hline & $\begin{array}{l}\text { Grupo } 1 \\
(n=25)\end{array}$ & $\begin{array}{l}\text { Grupo } 2 \\
(n=51)\end{array}$ & $\begin{array}{l}\text { Significación estadística } \\
\qquad(p<0,05)\end{array}$ \\
\hline Edad media & 65,4 & 61,4 & Ns \\
\hline $\begin{array}{l}\text { ASA } \\
1 \\
2 \\
3\end{array}$ & $\begin{array}{r}1 \\
7 \\
17\end{array}$ & $\begin{array}{r}1 \\
21 \\
29\end{array}$ & NS \\
\hline $\begin{array}{l}\text { Sexo } \\
\text { Hombre } \\
\text { Mujer }\end{array}$ & $\begin{array}{r}18 \\
7\end{array}$ & $\begin{array}{l}33 \\
18\end{array}$ & NS \\
\hline $\begin{array}{l}\text { Localización primario } \\
\text { Colon derecho } \\
\text { Colon izquierdo } \\
\text { Recto } \\
\text { Colon transverso } \\
\text { No consta (otro centro) }\end{array}$ & $\begin{array}{l}6 \\
5 \\
8 \\
6 \\
0\end{array}$ & $\begin{array}{r}9 \\
17 \\
13 \\
8 \\
4\end{array}$ & NS \\
\hline $\begin{array}{l}\text { Tamaño primario } \\
\mathrm{T} 1 / \mathrm{T} 2 \\
\mathrm{~T} 3 / \mathrm{T} 4 \\
\text { Invasión nodal } \\
\mathrm{N}- \\
\mathrm{N}+\end{array}$ & $\begin{array}{r}2 \\
23 \\
13 \\
12\end{array}$ & $\begin{array}{r}7 \\
40 \\
\\
10 \\
37\end{array}$ & $\begin{array}{c}\text { NS } \\
\mathbf{p}=\mathbf{0 , 0 2}\end{array}$ \\
\hline Número MTT (media) & 2 & 2,99 & $\mathrm{p}=0,05$ \\
\hline Tamaño medio MTT & $37,8 \mathrm{~mm}$ & $27 \mathrm{~mm}$ & NS \\
\hline CEA (valor medio) & 23,6 & 21,6 & NS \\
\hline Pacientes transfundidos & 10 & 25 & NS \\
\hline $\begin{array}{l}\text { Intervención } \\
\text { RH mayor vs RH menor } \\
\text { RH+RF } \\
\text { RF/OTROS } \\
\text { LE }\end{array}$ & $\begin{array}{c}3 \text { vs } 16 \\
3 \\
3 \\
0\end{array}$ & $\begin{array}{c}15 \text { vs } 12 \\
10 \\
10 \\
4\end{array}$ & $\begin{array}{c}\mathbf{p}=\mathbf{0 , 0 5} \\
\mathrm{NS} \\
\mathrm{NS} \\
-\end{array}$ \\
\hline Morbilidad (19,7\%) & $7(28 \%)$ & $8(15,6 \%)$ & NS \\
\hline Mortalidad (1,3\%) & $1(4 \%)$ & 0 & - \\
\hline Estancia hospitalaria & $13,8 \mathrm{~d}(8-32)$ & $11 d(4-19)$ & NS \\
\hline $\begin{array}{l}\text { Supervivencia } \\
1 \text { Año } \\
3 \text { Años } \\
5 \text { Años }\end{array}$ & $\begin{array}{l}75 \% \\
45 \% \\
36 \%\end{array}$ & $\begin{array}{l}76 \% \\
49 \% \\
29 \%\end{array}$ & NS \\
\hline Mediana supervivencia & $40,66 \mathrm{~m}$ & $34,05 \mathrm{~m}$ & NS \\
\hline
\end{tabular}

NS: Sin significación estadística. MTT: Metástasis. RH: Resección hepática. RF: Radiofrecuencia. LE: Laparotomía exploradora.

Tabla 2. Complicaciones según clasificación de Dindo y Clavien

\begin{tabular}{|rcccccc|}
\hline & Tipo I & Tipo II & Tipo IIIa & Tipo IIIb & Tipo IV & Total \\
\hline Grupo 1 & 2 & 4 & 0 & 1 & 0 & 7 \\
Grupo 2 & 0 & 6 & 1 & 0 & 1 & 8 \\
\hline
\end{tabular}



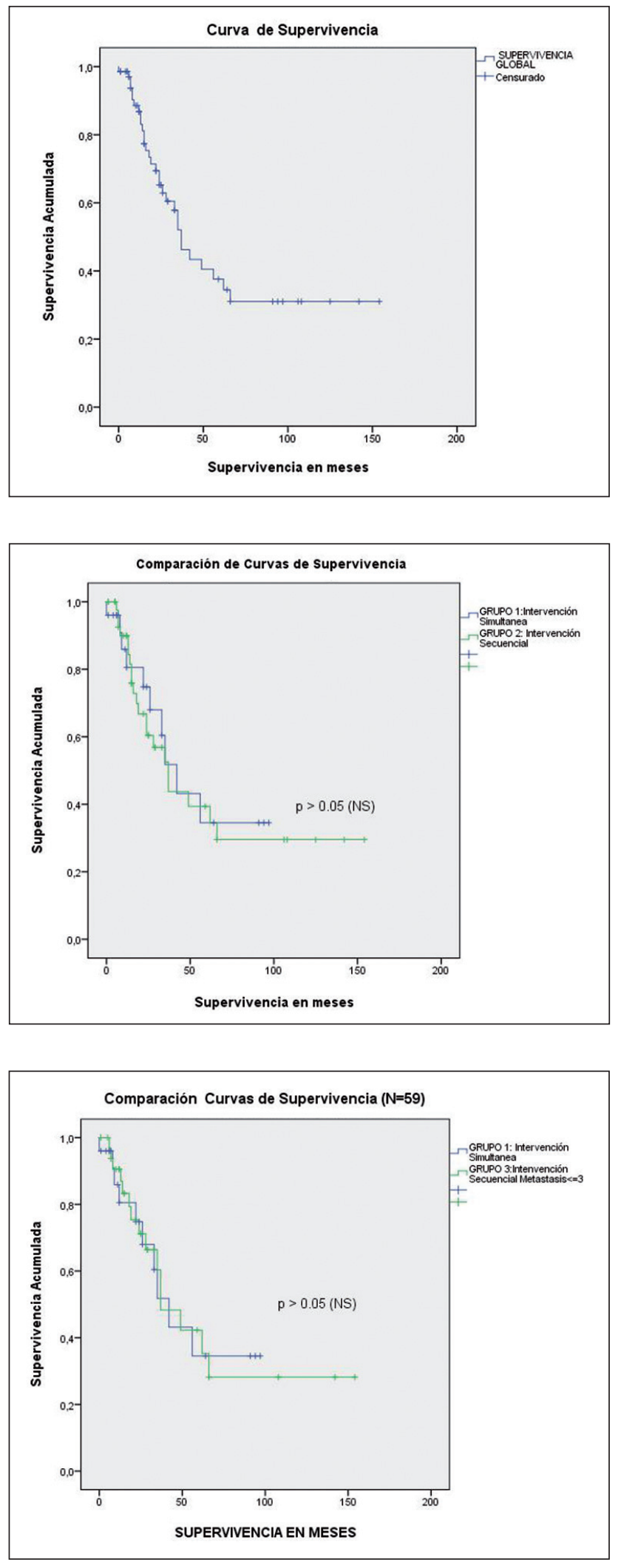

Figura 1. Curva de supervivencia global.

Figura 2. Supervivencia comparada grupos 1 y 2 .

Figura 3. Supervivencia comparada grupos 1 y 3 (aquellos del grupo 2 con un número de metástasis $<\mathrm{o}=3 ; \mathrm{n}=35$ ).

Rev Chil Cir. Vol 67 - № 2, Abril 2015; pág. 158-166 
en tanto que otros incluyen las que aparecen hasta 12 meses después, entendiendo que pasaron desapercibidas en el diagnóstico del tumor primario ${ }^{12}$. Nosotros las hemos definido como aquellas diagnosticadas antes o durante la intervención del primario, para poder comparar dos grupos más homogéneos como opinan otros autores ${ }^{11}$.

Existen varias estrategias para el tratamiento de las MHCRS: Clásicamente, se ha defendido la cirugía secuencial con resección del primario, quimioterapia y cirugía de las metástasis en pacientes con buena respuesta, ya que, aquellos sin respuesta a quimioterapia, tenían un mal pronóstico con o sin cirugía $^{13-15}$. Actualmente se está cuestionando esa estrategia en favor de una cirugía simultánea que ofrece un solo ingreso con menor coste global, el paciente va menos inmunodeprimido y con menor toxicidad hepática a la cirugía y hay menos posibilidad de diseminación tumoral $1^{5,7,12,16,17}$. Además, las posibles complicaciones tras la resección colorrectal podrían contribuir a retrasar el inicio de la quimioterapia y, por tanto, del abordaje de las $\mathrm{MH}$ en el caso de la cirugía secuencial ${ }^{5}$. La morbilidad era otro argumento a favor de la cirugía secuencial pero cada vez existen más publicaciones con similar morbilidad entre ambos procedimientos $5,6,7,17,18$.

Un tercer enfoque es el de "liver first" que consiste en QT seguida de resección hepática y, después de 8 semanas, cirugía del tumor primario; la desventaja principal es que en este período, el primario puede complicarse o progresar ${ }^{19,20}$. En el cáncer de recto, la neoadyuvancia en casos de T3, T4 y/o N+ es un índice de calidad en su manejo porque proporciona más resecciones $\mathrm{R} 0$, aumenta las tasas de conservación esfintérica y controla los síntomas pelvianos ${ }^{18}$. Sin embargo, en los casos T1, T2 y N0 donde no hay indicación de RQT neoadyuvante, la situación es la misma que para los de colon.

Ante todas estas estrategias, se ha intentado aclarar qué actitud es la mejor. Existen varios trabajos de revisiones ${ }^{17,21,23,24}$ y en todas ellas los autores encuentran los mismos problemas: 1) La definición de MHCRS no es la misma para todos los autores; 2) Son trabajos retrospectivos; 3) No siempre se comparan grupos con MHCRS y estrategias diferentes sino que son series de resultados con una $\mathrm{u}$ otra estrategia y 4) En general, los candidatos a cirugía simultánea son mejores que los de cirugía secuencial. Con estos datos, la mayoría coinciden en que la cirugía simultánea se puede realizar con los mismos resultados sólo en casos seleccionados, pero, en pacientes con peor status preoperatorio o candidatos a resecciones hepáticas mayores, la cirugía secuencial sería la indicada, con menor morbimortalidad y ahorrando posiblemente alguna intervención hepática innecesaria $^{15,17,23,24}$.
Entonces, para hacer una buena selección de los candidatos a una u otra estrategia, hay que responder a una serie de preguntas: ¿hay cirujanos expertos en ambas cirugías? ¿hay disponibilidad logística? ¿hay riesgo de complicaciones del tumor primario? ¿el paciente tiene comorbilidades que pueden agravarse con una cirugía más agresiva? ¿la localización del primario permite una cirugía simultánea? ¿hay extensión extrahepática? ¿se puede realizar una cirugía R0 del primario y de las MTT? ¿queda suficiente parénquima hepático sano después de la resección? En el caso del Ca de recto bajo: ¿qué esquema terapéutico en función de la extensión del primario y de las $\mathrm{MH}$ es el más idóneo?

Todas estas preguntas son de una gran complejidad y engloban problemas del paciente, del tumor primario, de las metástasis, del equipo quirúrgico, de la logística del centro hospitalario, etc. Deben ser contestadas y elegir la mejor estrategia de forma multidisciplinar e individualizando cada caso, siempre teniendo en cuenta que se debe intentar la resección $\mathrm{R} 0$ tanto en el primario como en las metástasis ${ }^{3}$.

En nuestro Centro todos los pacientes son tratados después de consensuar la estrategia en el comité oncoquirúrgico y esta cambió a partir de 2005, año en el que iniciamos la cirugía simultánea. El grupo de intervenciones secuenciales es mayor porque incluye pacientes intervenidos antes de esta fecha y aquellos procedentes de otros hospitales, con el primario ya resecado.

Los dos grupos son homogéneos en cuanto a edad, sexo, localización y riesgo anestésico (ASA) y similares a los de otras series ${ }^{12,20,22}$. El número de $\mathrm{MH}$ fue mayor en el grupo 2, algo esperable, ya que la cirugía simultánea la realizamos sólo en casos de 3 o menos metástasis hepáticas tal y como recomiendan la mayoría de los autores ${ }^{12,21,23,24}$. Por el mismo motivo el índice de resecabilidad fue mayor en el grupo 1 así como el porcentaje de resecciones hepáticas aunque sin significación estadística. Sin embargo, el número de resecciones mayores entre ambos grupos fue significativamente mayor en el grupo de cirugía secuencial. No siempre pudimos realizar cirugía con intención curativa; a pesar de que todos los pacientes tenían un estudio de extensión preoperatoria, encontramos discrepancias con los hallazgos intraoperatorios en 17 pacientes $(22,6 \%)$ lo que derivó en 13 casos de ablación por RF (en tres casos acompañada de embolización portal para una cirugía posterior) y 4 laparotomías exploradoras. Esto no es infrecuente y a pesar de la mejora de los medios diagnósticos, Elias et al., refieren en su revisión un $30 \%$ de laparotomías exploradoras, por encontrar hallazgos no esperados con las pruebas de imagen preoperatorias ${ }^{25}$. En pre- 
sencia de enfermedad extrahepática, aún cuando no es una contraindicación absoluta de resección de las metástasis (si se pueden resecar también las otras), los resultados son peores ${ }^{26}$, por lo que nosotros en esos casos, preferimos realizar radiofrecuencia y quimioterapia posterior. No tenemos experiencia en otras estrategias como el liver first.

El Índice de resecabilidad global fue de 77,6\%, menor que en otras series de nuestro entorno y tal vez por eso, unido a que las intervenciones hepáticas fueron realizadas por el mismo cirujano, la morbilidad global es menor y no hemos tenido complicaciones mayores ni diferencias entre ambos grupos $^{4,7,12,15,21-23,27,28}$ (Tabla 3), a pesar de que tenemos 46 pacientes ASA 3, por lo que no creemos que el status preoperatorio deba influir para elegir una $\mathrm{u}$ otra estrategia.

La supervivencia a 1, 3 y 5 años fue de $75 \%$, $45 \%$ y $36 \%$ en el grupo 1 y de $76 \%, 49 \%$ y $29 \%$ en el grupo 2, cifras similares a las encontradas en la literatura $^{5-7,12,15,20-24,29}$ (Tabla 3) sin diferencias significativas entre ambos grupos.

La debilidad de este estudio es que se trata de un análisis retrospectivo, aunque de una base prospectiva, y que los grupos no coinciden en el mismo espacio temporal. Por otra parte, los dos grupos son comparables en cuanto a las características epidemiológicas de los pacientes, localización del tumor primario e incluso riesgo anestésico pero no en lo que respecta a la invasión ganglionar y metastásica. El grupo de cirugía secuencial tiene un mayor número de metástasis y más resecciones mayores; podríamos esperar que este grupo tuviera más complicaciones postoperatorias y menor supervivencia; sin embargo, no observamos diferencias estadísticamente significativas. Por esto, quisimos comparar la supervivencia de los 25 pacientes de cirugía simultánea con aquellos de grupo 2 que podrían haberse beneficiado de la misma, por tener un número de $\mathrm{MH} \leq 3$, si hubieran sido de nuestro Hospital o se hubieran intervenido después de 2005 (a los que denominamos grupo 3); de este modo compararíamos dos grupos homogéneos pero tampoco encontramos diferencias significativas en la supervivencia (Figura 3).

A la vista de nuestros resultados y comparando con otras series, creemos que las MHCRS, pueden ser intervenidas de forma simultánea al tumor primario en pacientes con un número de $\mathrm{MH} \leq \mathrm{de} 3 \mathrm{y}$ de localización abordable por la misma incisión para ambos procedimientos, siempre que el equipo sea especializado. La morbimortalidad y la supervivencia son similares tanto en la intervención simultánea como en la secuencial. Serán los estudios comparativos prospectivos los que dicten la conducta a seguir en el futuro.

Tabla 3. Morbimortalidad y supervivencia actuarial de las series más recientes consultadas

\begin{tabular}{|c|c|c|c|c|c|}
\hline Autor & Año & $\begin{array}{l}\text { Resección: simultánea } \\
\text { Secuencial }\end{array}$ & Morbilidad & Mortalidad & $\begin{array}{c}\text { Supervivencia } 3-5 \\
\text { años }\end{array}$ \\
\hline Figueras (4) & 2001 & $\begin{array}{r}37 \\
213\end{array}$ & $\begin{array}{l}49 \% \\
35 \%\end{array}$ & $\begin{array}{l}9 \% \\
2,3 \%\end{array}$ & $60,1 \%-36,1 \%$ \\
\hline De Santibañes (6) & 2010 & 185 & $\begin{array}{c}21,08 \% \\
-\end{array}$ & $\begin{array}{l}1,08 \% \\
-\end{array}$ & - \\
\hline Martin (12) & 2009 & $\begin{array}{r}70 \\
160\end{array}$ & $\begin{array}{l}55 \% \\
56 \%\end{array}$ & $\begin{array}{l}2 \% \\
2 \%\end{array}$ & - \\
\hline Reddy (15) & 2009 & $\begin{array}{l}135 \\
475\end{array}$ & $\begin{array}{l}36 \% \\
18 \%\end{array}$ & $\begin{array}{l}1 \% \\
0,5 \%\end{array}$ & - \\
\hline Brouquet (20) & 2010 & $\begin{array}{l}43 \\
72\end{array}$ & $\begin{array}{l}47 \% \\
51 \%\end{array}$ & $\begin{array}{l}5 \% \\
3 \%\end{array}$ & $\begin{array}{l}65 \%-55 \% \\
58 \%-48 \%\end{array}$ \\
\hline Slupski (22) & 2009 & $\begin{array}{l}28 \\
61\end{array}$ & - & - & $\begin{array}{l}70 \%-45 \% \\
55 \%-38 \%\end{array}$ \\
\hline Capussotti (28) & 2007 & $\begin{array}{l}31 \\
48\end{array}$ & $\begin{array}{l}32,6 \% \\
56,3 \%\end{array}$ & $\begin{array}{l}3,2 \% \\
0\end{array}$ & - \\
\hline Yan (29) & 2007 & $\begin{array}{l}73 \\
30\end{array}$ & $\begin{array}{l}56 \% \\
67 \%\end{array}$ & $\begin{array}{l}0 \\
0\end{array}$ & $\begin{array}{l}53 \%-36 \% \\
49 \%-37 \%\end{array}$ \\
\hline Nuestra serie & 2013 & $\begin{array}{l}25 \\
51\end{array}$ & $\begin{array}{l}28 \% \\
15,6 \%\end{array}$ & $\begin{array}{l}4 \% \\
0\end{array}$ & $\begin{array}{l}45 \%-36 \% \\
49 \%-29 \%\end{array}$ \\
\hline
\end{tabular}




\section{Referencias}

1. Adam R, Hoti E, Bredt LC. Estrategias oncoquirúrgicas en el cáncer hepático metastático. Cir Esp. 2011;89:109.

2. Penna C. Prise en charge des patients ayant un cancer $\mathrm{du}$ foie. Les metastases hépatiques des cancers colorectaux. Bull Cancer 2003;90:79-83.

3. Minagawa M, Makuuchi M, Torzilli G, Takayama T, Kawasaki S, Kosuge T, et al. Extension of the frontiers of surgical indications in the treatment of liver metastases from colorectal cancer. Longterm results. Ann Surg. 2000;231:487-99.

4. Figueras J, Torras J, Valls C, Ramos E, Lama C, Busquets $\mathrm{J}$, et al. Resección de metástasis hepáticas de carcinoma colorrectal. Índice de resecabilidad y supervivencia a largo plazo. Cir Esp. 2001;70:27-33.

5. Hillings JG, Jorgensen PW. Staged or simultaneous resection of synchronous liver metastases from colorectal cancer. A systematic review. Colorectal Dis. 2008;2:310.

6. De Santibañes E, Fernández D, Vaccaro C, Quintana GO, Bonadeo F, Pekolj J, et al. Short-Term and LongTerm Outcomes after simultaneous resection of colorectal malignancies and synchronous liver metastases. World J Surg. 2010;34:2133-40.

7. De Santibañes E, Lassalle EB, McCormack L, Pekolj J, Quintana GO, Vaccaro C, et al. Simultaneous colorectal and hepatic resections for colorectal cancer: postoperative and longterm outcomes. J Am Coll Surg. 2002;195:196-202.

8. Strasberg SM. Nomenclature of hepatic anatomy and resection: a review of the Brisbane 2000 system. J Hepatobiliary Pancreat Surg. 2005;12:351-5.

9. Clavien PA, Barkun J, de Oliveira ML, Vauthey JN, Dindo D, Schulick RD, et al. The Clavien- Dindo classification of surgical complications: five-year experience. Ann Surg. 2009;250:187-96.

10. Martinez J, Jarufe N, González R, Álvarez M. Alternativas terapéuticas actuales de las metástasis hepáticas. Rev Med Chile 2008;136:376-84.

11. Yoshioka R, Hasegawa K, Mise Y, Oba M, Aoki T, Sakamoto Y, et al. Evaluation of the safety and efficacy of simultaneous resection of primary colorectal cancer and synchronous colorectal liver metastases. Surgery 2014:155:476-85.

12. Martin RC, Augenstein V, Reuter NP, Scoggins CR, McMasters KM. Simultaneous Versus Staged Resection for Synchronous Colorectal Cancer Liver Metastases. J Am Coll Surg. 2009;208:842-50.

13. Nordlinger B, Sorbye H, Glimelius B, Poston GJ, Schlag PM, Rougier P, et al. Perioperative chemotherapy with FOLFOX4 and surgery versus surgery alone for resectable liver metastases from colorectal cancer (EORTC Intergroup trial 40983): a randomised controlled trial. Lancet 2008;371:1007-16.
14. Adam R, Bhangui P, Poston G, Mirza D, Nuzzo G, Barroso E, et al. Is perioperative chemotherapy useful for solitary, metachronous, colorectal liver metastases? Ann Surg. 2010;252:774-87.

15. Reddy SK, Zorzi D, Lum YW, Barbas AS, Pawlik TM, Ribero D, et al. Timing of multimodality therapy for resectable synchronous colorectal liver metastases: a retrospective multi-institutional analysis. Ann Surg Oncol. 2009;16:1809-19.

16. Abbott DE, Cantor SB, Hu CY, Aloia TA, You YN, Nguyen $\mathrm{S}$, et al. Optimizing clinical and economic outcomes of surgical therapy for patients with colorectal cancer and synchronous liver metastases. J Am Coll Surg. 2012;215:262-70.

17. Mayo SC, Pulitano C, Marques H, Lamelas J, Wolfgang CL, de Saussure W, et al. Surgical management of patients with synchronous colorectal liver metástasis: A multicenter International analysis. J Am Coll Surg. 2013;216:707-16.

18. Dupré A, Gagnière J, Chen Y, Rivoire M. Prise en charge des métastases hépatiques synchrones d'origine colorectale. Bull Cancer 2013;100:363-71.

19. Mentha G, Roth AD, Terraz S, Giostra E, Gervaz P, Andres A, et al. "Liver first" approach in the treatment of colorectal cancer with synchronous liver metastases. Dig Surg. 2008;25:430-5.

20. Brouquet A, Mortenson MM, Vauthey JN, RodríguezBigas MA, Overman MJ, Chang GJ, et al. Surgical strategies for sinchronous colorectal liver metastases in 156 consecutive patients: classic, combined or reverse strategy. J Am Coll Surg. 2010;210:934-41.

21. Lycoudis PM, Reilly DO, Nastos K, Fusai G. Systematic review of surgical management of synchronous colorectal liver metastases. Br J Surg. 2014;101:605-12.

22. Slupski M, Wlodarczyk Z, Jasinski M, Masztalerz M, Tujakowski J. Outcomes of simultaneous and delayed resections of synchronous colorectal liver metastases. Can J Surg. 2009;52:241-4.

23. Slesser AAP, Simillis C, Goldin R, Brown G, Mudan $\mathrm{S}$, Tekkis PP. A meta-analysis comparing simultaneous versus delayed resections in patients with synchronous colorectal liver metastases. Surg Oncol. 2013;22:36-47.

24. Yin Z, Liu C, Chen Y, Bai Y, Shang C, Yin R, et al. Timing of Hepatectomy in Resectable Synchronous Colorectal Liver Metastases: Simultaneous or Delayed? Hepatology 2013;57:2346-57.

25. Elias D, Sidreis L, Pocard M, De Baere T, Dromain C, Lassau N, et al. Incidence of unsuspected and treatable metastatic disease associated with operable colorectal liver metastases discovered only at laparotomy (and not treated when performing percutaneous radiofrequency ablation). Ann Surg Oncol. 2005;12:298-302.

26. Ramia JM, Figueras J, de la Plaza R, García-Parreño. Resección de metástasis hepáticas en presencia de enfermedad extrahepática. Cir Esp. 2012;90:483-9.

27. Hu JJ, Zhou ZX, Liang JW, Wang Z, Zhou HT, Zhong 
B. PÉREZ-CABRERA y cols.

YX. Outcome analysis of simultaneous liver resection for synchronous liver metastases from colorectal cancer. Chin J Oncol. 2013;35:63-6.

28. Capussotti L, Ferrero A, Viganò L, Ribero D, Lo Tesoriere R, Polastri R. Major liver resections synchronous with colorectal surgery. Ann Surg Oncol. 2007;14:195201.

29. Yan TD, Chu F, Black D, King DW, Morris DL. Synchronous resection of colorectal primary cancer and liver metastases. World J Surg. 2007;31:1496-501. 